\title{
Application of an Artificial Neural Network for Predicting the Texture of Whey Protein Gel Induced by High Hydrostatic Pressure
}

\author{
Jinsong $\mathrm{He}^{1}$ and Taihua $\mathrm{Mu}^{2, *}$ \\ ${ }^{1}$ College of Biosystems Engineering and Food Science, Zhejiang University, \\ Hangzhou, 310058, China \\ hejinsong@mail.tsinghua.edu.cn \\ ${ }^{2}$ Institute of Agro-Food Science \& Technology, Chinese Academy of \\ Agricultural Sciences, Beijing 100094, China \\ mutaihua@126.com
}

\begin{abstract}
The effects of high hydrostatic pressure (HP), protein concentration, and sugar concentration on the gelation of a whey protein isolate (WPI) were investigated. Differing concentrations of WPI solution in the presence or absence of lactose $(0-20 \%, \mathrm{w} / \mathrm{v})$ were pressurized at $200-1000 \mathrm{MPa}$ and incubated at $30^{\circ} \mathrm{C}$ for $10 \mathrm{~min}$. The hardness and breaking stress of the HPinduced gels increased with increasing concentration of WPI (12-20\%) and pressure. Lactose decreased the hardness and breaking stress of the gel. Furthermore, these results were used to establish an artificial neural network (ANN). A multiple layer feed-forward ANN was also established to predict the physical properties of the gel based on the inputs of pressure, protein concentration, and sugar concentration. A useful prediction was possible, as measured by a low mean square error $(\mathrm{MSE}<0.05)$ and a regression coefficient $\left(R^{2}>0.99\right)$ between true and predicted data in all cases.
\end{abstract}

Keywords: Hydrostatic high pressure, Whey protein isolate, Gelation, Artificial neural network.

\section{Introduction}

Whey is a by-product of cheese manufacturing and contains about $13 \%$ protein by dry weight. Whey proteins possess outstanding physicochemical properties in gelation and binding, making them widely used as functional ingredients in many formulations of bakery, dairy, and sausage products [1]. The major constituents of milk whey protein are $\beta$-lactoglobulin $(\beta-\mathrm{Lg}), \alpha$-lactalbumin $(\alpha-\mathrm{La})$, bovine serum albumin (BSA), and immunoglobulins [2]. Five kinds of industrial whey proteins are currently produced, four of which have a whey protein concentration (WPC) classified into four grades according to the protein content $(35 \%, 50 \%, 65 \%$, and $70-90 \%)$. A whey protein isolate (WPI) contains $>90 \%$ protein on a dry weight basis [3].

\footnotetext{
* Corresponding author. 
One of the functional properties of whey proteins is gelation, which is induced by heat treatment [4-6], although other factors such as salt addition [7], acidification [8, 9], and enzyme treatment can cause gelation with or without heating [9-13]. Hydrostatic pressure has been shown to induce gelation in whey proteins under appropriate conditions [14-20]. The rheological properties of whey protein, i.e., storage modulus $G^{\prime}$ and loss modulus $G^{\prime \prime}$ [14], increase with increasing protein concentration. The gel strength also increases with increasing pressure holding time $[14,16,18]$. In addition, the contribution of intermolecular S-S bonds to the aggregation and gelation of whey proteins has been demonstrated by Tanaka et al. [21] and Kanno et al. [18].

In addition, artificial neural networks (ANNs) are among the most commonly used nonlinear techniques. An ANN is a mathematical algorithm whose structure and function is inspired by the organization and function of the human brain. The important property of a neural network is its ability to learn to improve its performance. ANN can handle nonlinear data and tend to produce lower prediction errors. ANN provides several advantages over conventional digital computations because of its faster data processing, learning ability and fault tolerance.

Controlling the texture of gel is important for the utilization of the gel in the food processing industry. In this context, new approaches are required to modulate and predict the physicochemical properties of the gel. In this present work, we studied the effect of pressure, protein concentration, and sugar concentration on the rheological properties of the pressure-induced gels formed from a WPI. The results should provide the basic information to modulate the texture of the gels by using the pressure, protein concentration, and sugar concentration as working parameters. Furthermore, a multilayer feed-forward neural network trained with an error backpropagation algorithm was employed to provide approaches to predict the physicochemical properties of the gel using these working parameters.

\section{Materials and Methods}

\subsection{Materials}

WPI powder from bovine milk was donated by the Central Research Institute of the Snow Brand Dairy Industry Co. (Saitama, Japan). This product contained $6.1 \%$ moisture, $89.8 \%$ protein, $1.8 \%$ ash, $1.3 \%$ lactose, and $0.5 \%$ lipids, and the fraction of individual whey protein was $74 \% \quad \beta-\mathrm{Lg}, 18 \% \quad \alpha-\mathrm{La}, 6 \% \quad \mathrm{BSA}$, and $2 \%$ immunoglobulins, according to the manufacturer. All the chemicals used were of analytical grade and were obtained from Wako Pure Chemical Industries (Osaka, Japan).

\subsection{Preparation of the WPI Solution}

A WPI solution (10-20\%, w/v) was prepared in a $50 \mathrm{mM}$ sodium phosphate buffer ( $\mathrm{pH}$ 6.8), the lactose being added to make a final concentration of $0-20 \%(\mathrm{w} / \mathrm{v})$. 
Each WPI solution was poured into a Teflon tube $(4 \mathrm{~mL}$ volume, $15 \mathrm{~mm}$ internal diameter and $22 \mathrm{~mm}$ depth) fitted with a screw cap and then subjected to $800 \mathrm{MPa}$ pressure with a HR15-B2 hand-operated oil-pressure generator (Hikari Koatsu, Hiroshima, Japan). The temperature was maintained at $30^{\circ} \mathrm{C}$. In each experiment, the indicated pressure was achieved within $1.5 \mathrm{~min}$, held for $10 \mathrm{~min}$, and then released to atmospheric pressure within $0.5 \mathrm{~min}$. The pressurized sample was analyzed within $1 \mathrm{~h}$ of releasing the pressure.

\subsection{Rheological Measurements}

The hardness and breaking stress of each gel sample was measured at room temperature with a Fudoh rheometer operated under RT-2005DD software (Rheotech, Tokyo, Japan) as described elsewhere [18].

\subsection{Modeling of the Neural Network}

The multilayer feed-forward neural network has proven to be an excellent universal approximator of non-linear functions. In this work, a feed-forward neural network trained with an error back-propagation algorithm was employed using MATLAB (Version 2007a, Mathworks, Natick, MA). The Neural Network Toolbox was used to model the hardness and breaking stress of the gel. The input parameters chosen in this study were pressure, WPI concentration, and sugar concentration. Supervised learning was used to train this network. The predicted and desired output were compared with one another while the errors were calculated between the predicted and actual output. An error back-propagation algorithm was used to adjust the network weights. It used a Levenberg-Marquardt approach, in which the weights were changed in proportion to the value of the error gradient. The training iterations were stopped when the validation error reached a set minimum.

\subsection{Statistical Analysis}

Statistical analysis was performed using the package DeltaGraph, Version 5 for the Mac (SPSS Inc., USA). In order to visualize the results of multiple experiments, the average and the standard deviation were calculated.

\section{Results and Discussion}

\subsection{Effects of Pressure and Protein Concentration}

The WPI solution at a concentration of $10-16 \%$ formed a gel at $600 \mathrm{MPa}$, while the $20 \%$ WPI solution formed a gel at $400 \mathrm{MPa}$. The gels formed from the $20 \% \mathrm{WPI}$ solution at $400 \mathrm{MPa}$ and from the $16 \%$ solution at $600 \mathrm{MPa}$ were soft and translucent in appearance. 
The gels formed from 10\% WPI solution under $600 \mathrm{MPa}$, and 12\% WPI solution under $400 \mathrm{MPa}$ were too soft to measure texture properties using the rheometer.

Figure 1 shows the hardness and breaking stress of those gels that were sufficiently firm formed from 12 to $20 \%$ WPI at different pressures. The hardness and breaking stress of the WPI gels increased with WPI concentration, increasing from 12 to $18 \%$ at a constant pressure and, similarly, those of each solution at a constant concentration rose as hydrostatic pressure was increased from 600 to $1000 \mathrm{MPa}$. In addition, no water was expelled from the gel matrix that had been induced from these WPI samples under pressure during the measurement of hardness and breaking stress.

The gel-forming ability of a protein depends on a critical balance between the attractive and repulsive forces of the protein molecules [22]. Hydrogen bonds, electrostatic bonds, hydrophobic interaction, and intermolecular disulfide linkages may play a role in gel formation in protein solutions $[22,23]$. Under high pressure, $\beta$ Lg unfolds, resulting in the exposure of its free SH group [21, 24]. Unfolded $\beta$-Lg can interact, through SH/SS interchange reactions, with proteins containing disulfide bonds, e.g. $\alpha$-La, BSA, and $\beta-\operatorname{Lg}[19,25]$. Regarding pressure-induced gelation of a WPI solution, the formation of intermolecular S-S bonds between proteins has been confirmed $[18,25]$, and it is likely that the gel is mainly formed by the cross-linking of intermolecular S-S bonds [25].
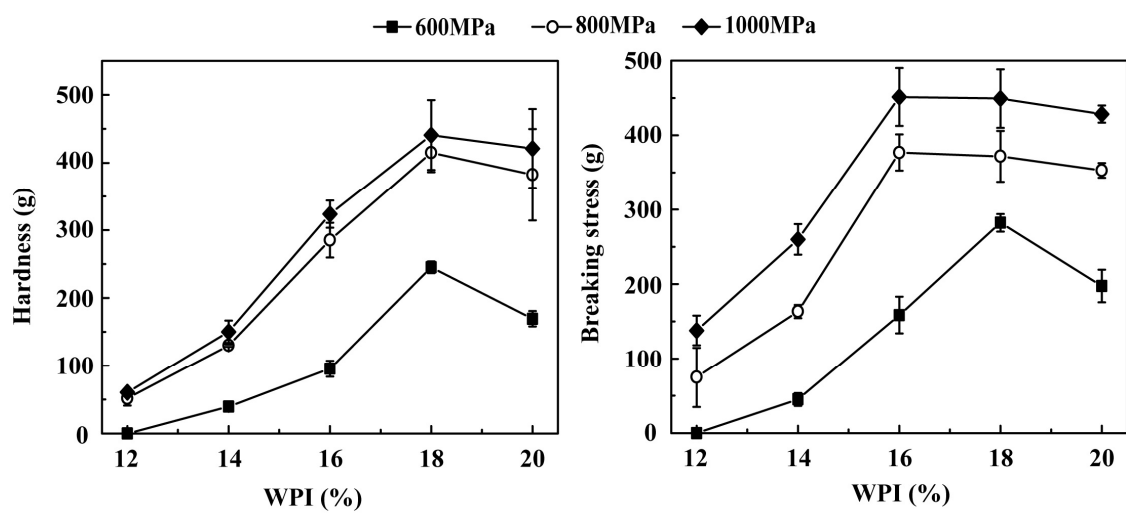

Fig. 1. Effect of pressure and WPI concentration on the hardness, and breaking stress of pressure-induced gels from WPI. WPI $(12 \sim 20 \%$, w/v) was pressurized at 600,800 , or 1000 $\mathrm{MPa}$ and $30^{\circ} \mathrm{C}$ for $10 \mathrm{~min}$. Bars show the standard deviation for three measurements with different gel samples.

\subsection{Effects of Sugar Concentration}

To study the effect of the sugar concentration (2\% to $20 \%$ ) (w/v) on gels prepared from WPI in a phosphate buffer by pressurizing at $800 \mathrm{MPa}$ and $30^{\circ} \mathrm{C}$ for $10 \mathrm{~min}$, the lactose content, the main sugar contained in bovine milk, was varied. Figure 2 shows the changes in rheological properties as a function of the lactose content. The 
hardness and breaking stress of the gel decreased with increasing concentration of lactose. This indicated that the sugars weakened the intermolecular interactions of the protein, seemingly preventing the cross-linking between proteins.

Sugar is preferentially excluded from the protein domain in an aqueous mixture of protein and sugar. This exclusion effect of the sugar can affect the surface tension of the water and minimize the protein-solvent interface [17, 26, 27]. As a result, the sugar protected the protein from unfolding and subsequent aggregation under pressure [28]. Sugars can also stabilize a protein against pressure-induced denaturation [17, 28]. The presence of $5 \%$ sucrose in $2.5 \% \beta-\mathrm{Lg}$ decreased the protein unfolding, slightly increasing the rate of reversibility of aggregates by pressurization at $450 \mathrm{MPa}$ [28]. The gelation of protein is influenced in a complex manner by the processes of protein denaturation and aggregation [22, 29]. Sugars decrease the degree of intermolecular S-S bonding of proteins [25], and restrain phase separation during the gelation of WPI under high pressure.

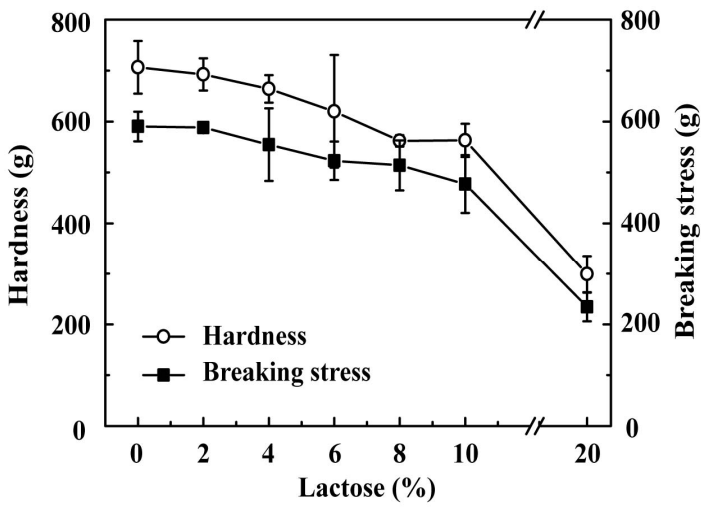

Fig. 2. Effect of lactose concentration on the hardness and breaking stress of a pressure-induced gel from WPI. WPI $(20 \%, \mathrm{w} / \mathrm{v})$ and the indicated concentration of lactose were dissolved in a $50 \mathrm{mM}$ sodium phosphate buffer at $\mathrm{pH} 6.8$, and the mixture was pressurized at $800 \mathrm{MPa}$ and $30^{\circ} \mathrm{C}$ for $10 \mathrm{~min}$. Bars show the standard deviation for three measurements with different gel samples.

\subsection{Evaluation of Model Predictability}

The ratio of the explained variation to the total variation, $R^{2}$, reflects the degree of fit for the mathematical model. The closer the value is to 1 , the better the model fits the actual data

$$
R^{2}=1-\frac{\sum_{i-1}^{n}\left(y_{i}-y_{d i}\right)^{2}}{\sum_{i-1}^{n}\left(y_{d i}-y_{m}\right)^{2}}
$$

where $\mathrm{n}$ is the number of points, $y_{\mathrm{i}}$ is the predicted value obtained from the neural network model, $y_{\mathrm{di}}$ is the actual value, and $y_{\mathrm{m}}$ is the average of the actual values. 
MSE is another important index to show the degree of fit of the model. It is calculated using the following equation.

$$
M S E=\frac{1}{n} \sum_{i=1}^{n}\left(y_{i}-y_{d i}\right)^{2}
$$

In this work, the range of three independent variables (pressure, WPI concentration, and sugar concentration) for building the neural network was set. The input matrix and the properties of gel are shown in Figs. 1 and 2. To understand the generalization capacity of the network, 19 input values were divided into three sets: 13 values for the training set and three values each for the validation and testing set. Figure 3 shows the plot of the predicted values and actual values of the hardness and breaking stress of the gels as well as $R^{2}$ and MSE. The trained network gave a $R^{2}$ of 0.998 and a MSE of 0.047 . The $R^{2}$ values of the training, the validation and the testing sets were 0.998 , 0.999 and 0.999, while the MSE values of the three sets were 0.047, 0.042 and 0.045, respectively. The network could predict the properties of the gel within a range of $\pm 7.8 \%$ of the actual value. The $R^{2}$, MSE and prediction range indicated a good agreement between the predicted value of the neural network model and the actual value, which also confirmed good generalization of the network.
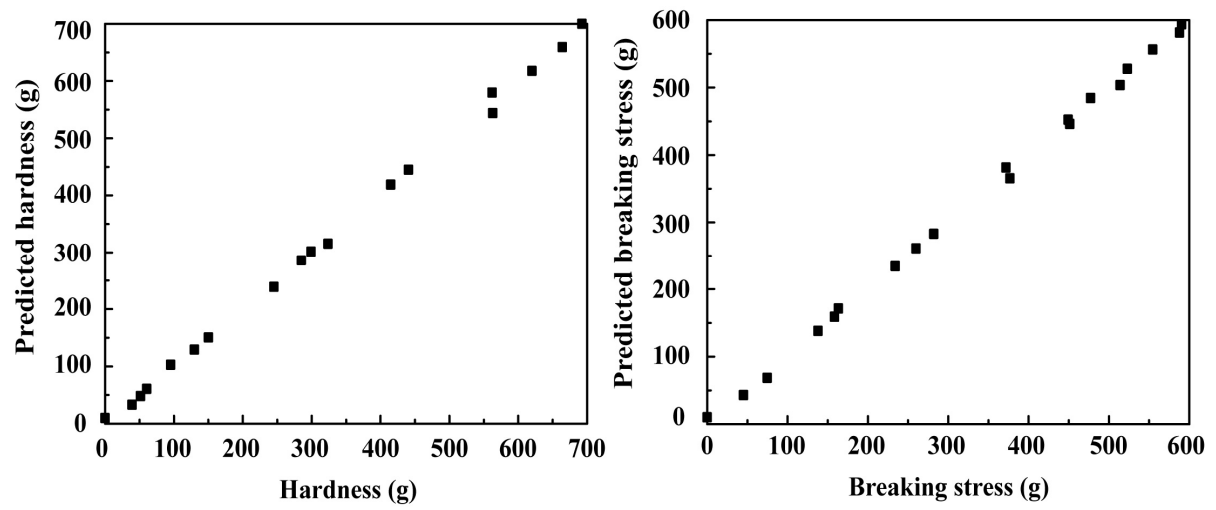

Fig. 3. Correlation between the predicted values of the neural network and the actual values for the hardness and breaking stress of a pressure-induced gel from WPI. The input matrix and the properties of gel as the output matrix are shown in Figs. 1 and 2.

\section{Conclusion}

The hardness and breaking stress of pressure-induced gels from WPI increased with increasing WPI concentration (12-18\%) and hydrostatic pressure, and decreased with increasing lactose concentration (0-20\%). The ANN provided a model to search for the non-linear nature between induced conditions and rheological properties in an efficient manner. The trained network gave an R2 value of 0.999 and an MSE value of 0.047 , which implied a good agreement between the predicted values and the actual values for the hardness and breaking stress of the gels, and confirmed good generalization of the network. 
Acknowledgments. The project was supported by the Zhejiang Key Scientific and Technological Innovation Team Project (2009R50001), the Department of Education of Zhejiang Province (Y201018520), and the Fundamental Research Funds for the Central Universities.

\section{References}

1. Kinsella, J.E., Whitehead, D.M.: Proteins in whey: chemical, physical, and functional properties. Adv. Food Nutri. Rese. 33, 343-438 (1989)

2. Eigel, W.N., Butler, J.E., Ernstrom, C.A., Farrell, H.M., Harwalkar, V.R., Jenness, R., Whitney, R.M.: Nomenclature of proteins of cow's milk: fifth revision. J. Dairy Sci. 67, 1599-1631 (1984)

3. Mulvihill, D.M.: Production, functional properties and utilization of milk protein products. In: Fox, P.F. (ed.) Advanced Dairy Chemistry, vol. 1, pp. 369-402. Elsevier Applied Science, London (1994)

4. Mulvihill, D.M., Donovan, M.: Whey proteins and their thermal denaturation-a review. Irish J. Food Sci. Technol. 11, 43-75 (1987)

5. Mulvihill, D.M., Kinsella, J.E.: Gelation characteristics of whey proteins and $\beta$-lactoglobulin. Food Technol. 41, 102-111 (1987)

6. Errington, A.D., Foegeding, E.A.: Factors determining fracture stress and strain of finestranded whey protein gels. J. Agric. Food Chem. 46, 2963-2967 (1998)

7. Barbut, S., Foegeding, E.A.: Calcium-induced gelation of preheated whey protein isolate. J. Food Sci. 58, 867-871 (1993)

8. Mleko, S., Foegeding, E.A.: pH induced aggregation and weak gel formation of whey protein polymers. J. Food Sci. 65, 139-143 (2000)

9. Britten, M., Giroux, H.J.: Acid-induced gelation of whey protein polymers: effects of $\mathrm{pH}$ and calcium concentration during polymerization. Food Hydrocoll 15, 609-617 (2001)

10. Faergemand, M., Qvist, K.B.: Transglutaminase: effect on rheological properties, microstructure and permeability of set style acid skim milk gel. Food Hydrocoll 11, 287-292 (1997)

11. Faergemand, M., Murray, B.S., Dickinson, E.: Cross-linking of milk proteins with transglutaminase at the oil-water interface. J. Agric. Food Chem. 45, 2514-2519 (1997)

12. Wilcox, C.P., Clare, D.A., Valentine, V.W., Swaisgood, H.E.: Immobilization and utilization of the recombinant fusion proteins trypsin-streptavidin and streptavidintransglutaminase for modification of whey protein isolate functionality. J. Agric. Food Chem. 50, 3723-3730 (2002)

13. Wilcox, C.P., Swaisgood, H.E.: Modification of the rheological properties of whey protein isolate through the use of an immobilized microbial transglutaminase. J. Agric. Food Chem. 50, 5546-5551 (2002)

14. Van Camp, J., Huyghebaert, A.: A comparative rheological study of heat and high pressure induced whey protein gels. Food Chem. 54, 357-364 (1995)

15. Van Camp, J., Huyghebaert, A.: High pressure-induced gel formation of a whey protein and haemoglobin protein concentrate. L.-Wiss. und-Technol. 28, 111-117 (1995)

16. Van Camp, J., Feys, G., Huyghebaert, A.: High-pressure-induced gel formation of haemoglobin and whey proteins at elevated temperatures. L.-Wiss. und-Technol. 29, 49-57 (1996) 
17. Dumay, E.M., Kalichevsky, M.T., Cheftel, J.C.: Characteristics of pressure-induced gels of $\beta$-lactoglobulin at various times after pressure release. L.-Wiss. und-Technol. 31, 10-19 (1998)

18. Kanno, C., Mu, T.-H., Hagiwara, T., Ametani, M., Azuma, N.: Gel formation from industrial milk whey proteins under hydrostatic pressure: effect of hydrostatic pressure and protein concentration. J. Agric. Food Chem. 46, 417-424 (1998)

19. Kanno, C., Mu, T.-H.: Gel formation of individual milk whey proteins under hydrostatic pressure. In: Hayashi, R. (ed.) Trends in High Pressure Bioscience and Biotechnology, pp. 453-460. Elsevier Science B.V. (2002)

20. Keenan, R.D., Young, D.J., Tier, C.M., Jones, A.D., Underdown, J.: Mechanism of pressure-induced gelation of milk. J. Agric. Food Chem. 49, 3394-3402 (2001)

21. Tanaka, N., Tsurui, Y., Kobayashi, I., Kunugi, S.: Modification of the single unpaired sulfhydryl group of beta-lactoglobulin under high pressure and the role of intermolecular S-S exchange in the pressure denaturation. Inter. J. Biolog. Macromol. 19, 63-68 (1996)

22. Ziegler, G.R., Foegeding, E.A.: The gelation of proteins. Adv. Food Nutr. Res. 34, 203-298 (1990)

23. Clark, A.H., Ross-Murphy, S.B.: Structural and mechanical properties of biopolymer gels. Adv. Poly. Sci. 83, 57-192 (1987)

24. Botelho, M.M., Valente-Mesquita, V.L., Oliveira, K.M., Polikarpov, I., Ferreira, S.T.: Pressure denaturation of beta-lactoglobulin: Different stabilities of isoforms A and B, and an investigation of the Tanford transition. FEBS 267, 2235-2241 (2000)

25. He, J.-S., Azuma, N., Hagiwara, T., Kanno, C.: Effects of sugars on the cross-linking formation and phase separation of high pressure induced gel of whey protein from bovine milk. Biosci. Biotechno. Biochem. 70, 615-625 (2006)

26. Timasheff, S.N.: The control of protein stability and association by weak interactions with water: how do solvents affect these processes? Annu. Rev. Biophys. Biomol. Struct. 22, 67-97 (1993)

27. Lee, J.C., Timasheff, S.N.: The stabilization of proteins by sucrose. J. Biol. Chem. 256, 7193-7201 (1981)

28. Dumay, E.M., Kalichevsky, M.T., Cheftel, J.C.: High-pressure unfolding and aggregation of $\beta$-lactoglobulin and the baroprotective effects of sucrose. J. Agric. Food Chem. 42, 1861-1868 (1994)

29. Van Camp, J., Messens, W., Clément, J., Huyghebaert, A.: Influence of pH and sodium chloride on the high pressure-induced gel formation of a whey protein concentrate. Food Chem. 60, 417-424 (1997) 